\title{
Design and Methodology for Technical Textiles
}

\section{Tamer F Khalifa*}

Department of Spinning, Weaving and Knitting, Faculty of Applied Arts, Helwan University, Egypt

Nowadays, the application of textiles are being continuously expanded in several technical application fields starting from its use in agriculture, military, structural, telecommunications, aerospace sector to very high sensitive medical applications, and etc. The specific and critical character of the technical applications imposed a dynamic change in the fields of the design, engineering, production and testing. The traditional empirical approach has been replaced by the careful modeling, calculation of the properties, prediction of the behavior and the final evaluation of the performance. The new technological position and role of the textiles causes important changes and evolutions in some key fields. The use of intelligent materials which are capable of reacting to external stimuli is growing in the field of textiles.

Production of technical textiles is a rapidly developing trade in the textile industry, due to availability of the new material technologies, for example, high modulus and high tenacity fibres (HMHT), heat-storage and thermo-regulated materials, Phase Change Materials (PCM), Shape Memory Materials (SMM), conductive polymers, micro-capsules and nano-materials.

New textiles developments, high performance material applications, creative structures, new production process and techniques are coming fast and furious, offering the possibilities of replacing metal and plastic with stronger, lighter and often cheaper alternatives. These innovations in textile materials have opened a range of possibilities for the engineers and designers to explore a wide range of technical products, such as reinforcements in composites for aerospace or marine applications or textiles for medical applications, defense, geotextiles, filtration, ballistic-proof etc. (Figure 1). Thus the design, methodology, concept
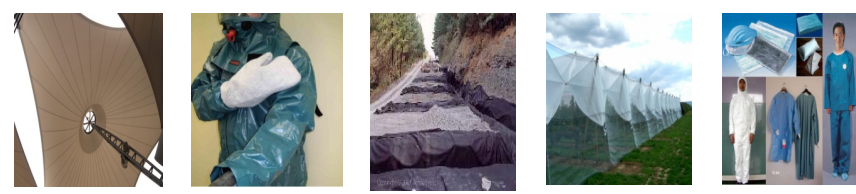

Figure 1: Technical textiles fields of applications.

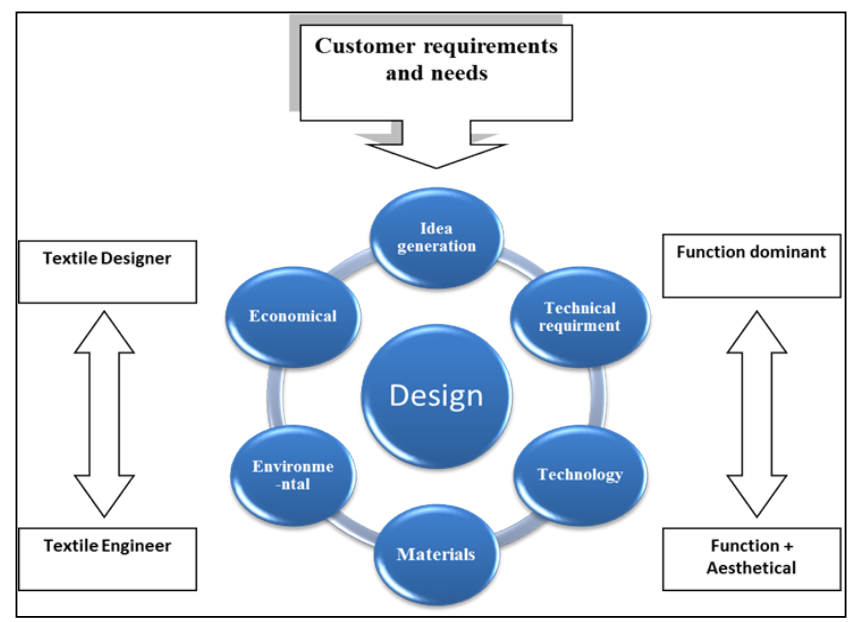

Figure 2: Technical textile design spectrum. and prediction of the end-product's mechanical properties are of major importance.

However, technical textile design innovation in addition to adapting new technologies, information, and knowledge are set to be the driving forces within the industry for fulfilling market needs, concerned with addressing customer needs; performance, quality, ergonomics, form and aesthesis in addition to economic aspects.

Design is the act of formalizing and transforming an idea or concept into tangible formation. It is distinct from making or building, a complex oriented process (Figure 2).

Meanwhile, the complexity of technical textile design, is the

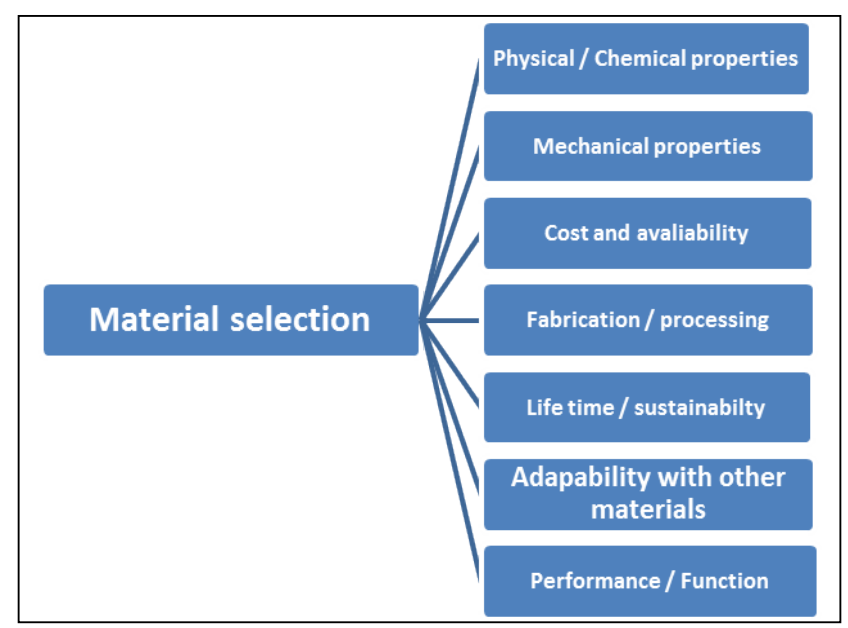

Figure 3: Factors affecting material selection for design process.

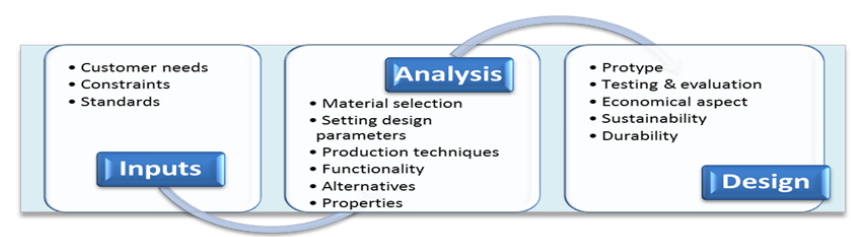

Figure 4: Design model analysis.

*Corresponding author: Tamer F Khalifa, Department of Spinning, Weaving and Knitting, Faculty of Applied Arts, Helwan University, Egypt, Tel: 0122-3824643; E-mail: tamerkhalifa@hotmail.com

Received November 06, 2013; Accepted November 11, 2013; Published November 18, 2013

Citation: Khalifa TF (2013) Design and Methodology for Technical Textiles. J Textile Sci Eng 3: e117. doi:10.4172/2165-8064.1000e117

Copyright: (c) 2013 Khalifa TF. This is an open-access article distributed under the terms of the Creative Commons Attribution License, which permits unrestricted use, distribution, and reproduction in any medium, provided the original author and source are credited. 
merging of different components (materials) in the same design like in composites and hybrid textiles, where different materials base issued; fibers, resins, ceramics, metals etc. (Figure 3) and the diversity of their properties and characterization offer a design challenge on the way of altering the conventional application of textiles.

Technical textile design is a structured engineered creative process where one of the major challenges for technical textile designers is the compromise of all these needs, parameters, and requirements in a design that fulfill the purpose. Therefore establishing a design framework (illustrating design processing) is set to be an essential and important design tool, not only this but a design matrix, addressing all requirements with respect to design parameters. Through assigning the design goals, functions and requirements, which then are translated to properties and performance needs, upon which designers begin setting design elements (materials, structures, techniques, production process, alternative materials) through a creative process (Figure 4) where in many cases it is addressed as tailored design (upon needs for special applications).

Modern technical textiles are indispensable material and tools for science and technology. Technical Textiles are set to be a promising field, offering a multifunctional material and a structure achieving high levels of physical, mechanical, thermal and chemical properties through an engineered design process based on scientific design and technology concepts. Technical Textiles offer the "best material of the world": lightest, strongest, biocompatible, smart, even intelligent. 